Research

\title{
Developing and evaluating stereotactic lung RT trials: what we should know about the influence of inhomogeneity corrections on dose
}

\author{
Danny Schuring* and Coen W Hurkmans
}

Address: Catharina-hospital, Department of radiotherapy, Michelangelolaan 2, P.O box 1350, 5602 ZA, Eindhoven, The Netherlands

Email: Danny Schuring* - danny.schuring@cze.nl; Coen W Hurkmans - coen.hurkmans@cze.nl

* Corresponding author

Published: 28 July 2008

Radiation Oncology 2008, 3:21 doi:10.1186/1748-717X-3-21

This article is available from: http://www.ro-journal.com/content/3/I/2I

(C) 2008 Schuring and Hurkmans; licensee BioMed Central Ltd.

This is an Open Access article distributed under the terms of the Creative Commons Attribution License (http://creativecommons.org/licenses/by/2.0), which permits unrestricted use, distribution, and reproduction in any medium, provided the original work is properly cited.

\begin{abstract}
Purpose: To investigate the influence of inhomogeneity corrections on stereotactic treatment plans for non-small cell lung cancer and determine the dose delivered to the PTV and OARs.

Materials and methods: For 26 patients with stage-I NSCLC treatment plans were optimized with unit density (UD), an equivalent pathlength algorithm (EPL), and a collapsed-cone (CC) algorithm, prescribing $60 \mathrm{~Gy}$ to the PTV. After optimization the first two plans were recalculated with the more accurate CC algorithm. Dose parameters were compared for the three different optimized plans. Dose to the target and OARs was evaluated for the recalculated plans and compared with the planned values.
\end{abstract}

Results: For the CC algorithm dose constraints for the ratio of the $50 \%$ isodose volume and the PTV, and the $\mathrm{V}_{20} \mathrm{~Gy}$ are harder to fulfill. After recalculation of the UD and EPL plans large variations in the dose to the PTV were observed. For the unit density plans, the dose to the PTV varied from 42.I to $63.4 \mathrm{~Gy}$ for individual patients. The EPL plans all overestimated the PTV dose (average 48.0 Gy). For the lungs, the recalculated $V_{20}$ Gy was highly correlated to the planned value, and was $12 \%$ higher for the UD plans $\left(R^{2}=0.99\right)$, and $15 \%$ lower for the EPL plans $\left(R^{2}=0.96\right)$.

Conclusion: Inhomogeneity corrections have a large influence on the dose delivered to the PTV and OARs for SBRT of lung tumors. A simple rescaling of the dose to the PTV is not possible, implicating that accurate dose calculations are necessary for these treatment plans in order to prevent large discrepancies between planned and actually delivered doses to individual patients.

\section{Introduction}

Treatment outcome of conventional radiotherapy for early-stage lung cancer has been rather poor, while possibilities for dose escalation are limited. In recent years several studies have shown promising results using stereotactic body radiotherapy (SBRT) for lung tumors, with local control rates at 3 years up to $90 \%$ [1-3].
A wide variety of treatment planning algorithms is used for SBRT. As a result, large differences exist in the way that inhomogeneities in the target volume are handled in the planning phase. In two important SBRT of lung cancer trials, on which many current clinical implementations of SBRT are based, different algorithms were used; the RTOG 0236 phase-II trial planning was performed without using 
inhomogeneity corrections assuming the patient has unit density [4], while in the Japanese JCOG 0403 trial a wide variety of inhomogeneity correction algorithms were allowed [5].

Planning algorithms can roughly be separated in (a) ones which do not take into account changes in lateral electron transport (pencil beam-like algorithms) and (b) ones that do take into account these changes (convolution-superposition type algorithms) [6]. In the type-a algorithms the effects of inhomogeneities are accounted for by applying a correction based on equivalent pathlength (EPL), like the Batho or ETAR correction. In the type-b algorithms changes in the lateral transport are modeled in an approximate way, and several studies have shown these algorithms to be more accurate for dose calculations in regions with inhomogeneities $[7,5,8]$. In particular, the collapsedcone convolution-superposition algorithm in most cases shows satisfactory agreement with Monte Carlo simulations in the case of inhomogeneous targets $[9,10]$. The Monte Carlo algorithms can be seen as the current gold standard for these types of dose calculations.

Several authors have studied the influence of inhomogeneity corrections on dose distributions specifically for stereotactic treatments of lung cancer [7,11-14]. Compared to conventional radiotherapy larger deviations can be expected due to the small field sizes used for treating these tumors. Most studies concentrated on creating a treatment plan using a type-a pencil beam algorithm, and recalculating the plans with a type-b algorithm or using Monte Carlo simulations, mostly for a small number of patients with relatively large tumors or on a phantom. All these studies have shown a significant overestimation of the target dose when using pencil-beam calculations.

In this study, the influence of inhomogeneity corrections on the dose distributions was investigated for a large group of patients with small stage I lung cancer tumors. These results are e.g., important for the correct interpretation of previous clinical trials and for the definition of planning criteria for new clinical trials of this treatment, and are being used for designing a Dutch multicenter randomized phase-III trial comparing SBRT with surgery for stage-I NSCLC (ROSEL trial).

\section{Materials and methods}

\section{Respiration-correlated CT and target delineation}

All patients in this study received a respiration-correlated 4D-CT using a Philips Brilliance Big Bore CT prior to treatment. The 4D-CT was reconstructed in ten equally spaced time bins using phase binning. From these phases, a maximum intensity projection (MIP) was reconstructed. The datasets were then imported in the Pinnacle ${ }^{3}$ treatment planning system (Philips Medical Systems, Wisconsin).
Using the MIP dataset, an experienced radiation oncologist delineated the internal target volume (ITV). The GTV was delineated on the CT dataset of the maximum inhale phase; tumor mobility was determined by translating the delineated GTV from this phase to the maximum exhale phase. Organs at risk were delineated on an average-density CT reconstruction. As dictated by the RTOG 0236 protocol, no ITV to CTV margin was applied [4]. The PTV was created by expanding the ITV with a $3 \mathrm{~mm}$ margin to account for setup uncertainties in accordance with the protocol used in by Lagerwaard et al [3].

\section{Patient characteristics}

Twentysix consecutive patients with non-small cell lung cancer (NSCLC) were included. All patients had solitary stage-I tumors, were medically inoperable and were treated at our institute with SBRT. A summary of the volumetric and motion characteristics of these tumors is shown in Table 1. The median, 25\%, 50\%, 75\% and $100 \%$ percentile values for the PTV were $29.4,15.8,29.4$, 40.2 , and $107.6 \mathrm{~cm}^{3}$, respectively.

\section{Treatment planning}

The treatment plans consisted of 9 equally spaced coplanar $6 \mathrm{MV}$ beams. Beams consisting of 2 segments were not allowed to enter through the esophagus, heart, spinal cord or contralateral lung. The plans were inversely optimized using the direct aperture optimization module of the Pinnacle ${ }^{3}$ treatment planning system. Dose calculations were performed on an average-density CT using a $3 \times 3 \times$ $3 \mathrm{~cm}$ dose calculation grid size.

Plans were optimized until $95 \%$ of the PTV received the prescription dose of 60 Gy in 3 fractions (according to RTOG 0236), and more than 99\% of the PTV received $90 \%$ of the prescribed dose ( $54 \mathrm{~Gy}$ ). No limitations to the maximum dose were applied within the PTV as highly inhomogeneous dose distributions are commonly accepted in stereotactic treatments. Objectives were added to ensure that the prescription isodose closely conforms to the PTV and the dose to healthy lung tissue was minimized. The goal was to keep the fraction of healthy lung receiving more than $20 \mathrm{~Gy}\left(\mathrm{~V}_{20 \mathrm{~Gy}}\right)$ below $10 \%$. For the $\mathrm{V}_{20}$ Gy' both lungs minus the ITV were delineated, in accordance with the RTOG protocol. Maximum dose to the spinal cord was limited to $18 \mathrm{~Gy}$, to the esophagus to $27 \mathrm{~Gy}$, and to the heart to $30 \mathrm{~Gy}$. To prevent the generation of very small segments the minimum beam segment area was set to $4 \mathrm{~cm}^{2}$, but generally the segment area was significantly larger. The minimum number of monitor units per segment was limited to $50 \mathrm{MU}$ to ensure that the delivery time at least covers one breathing cycle. All plans consisted of 9 beams with in total generally 18 segments and in a few cases 17 segments. 
Table I: Patient characteristics of the group of 26 patients used in this study.

\begin{tabular}{lc}
\hline Characteristics & Mean value (range) \\
\hline GTV $\left(\mathrm{cm}^{3}\right)$ & $13.9[0.7-50.4]$ \\
ITV $\left(\mathrm{cm}^{3}\right)$ & $19.3[1.4-64.1]$ \\
PTV $\left(\mathrm{cm}^{3}\right)$ & $37.3[5.5-107.6]$ \\
$V_{\text {ITV }} / V_{\text {GTV }}$ & $1.6[1.0-2.7]$ \\
Peak-to-peak amplitude $(\mathrm{cm})$ & $0.8[0.0-3.0]$ \\
Lung volume $\left(\mathrm{cm}^{3}\right)$ & $4942[2720-8786]$ \\
\hline
\end{tabular}

Three different plans were created. The first treatment plan was optimized using full inhomogeneity corrections using the CC algorithm. A second plan was created assuming all tissues within the body to have unit density (UD), in accordance with the RTOG 0236 protocol. The third plan was optimized while only accounting for the decreased attenuation of the primary photons, thus resembling an equivalent pathlength (EPL) correction that is incorporated in less advanced dose calculation algorithms. These plans will be referred to as the CC, UD and EPL plans respectively. For all patients, these three plans were optimized until all planning criteria were met. A small renormalization was applied to all plans to ensure that they had exactly identical PTV coverage (60 Gy to $95 \%$ of the PTV). Next, the UD and EPL plans were copied and recalculated without re-optimization using the collapsed-cone algorithm.

For each plan the maximum, minimum and mean dose to the PTV was determined, as well as the dose received by $95 \%\left(D_{95}\right)$ and $99 \%\left(D_{99}\right)$ of the PTV, and the isocenter dose. Conformality of the PTV coverage was evaluated by the ratio of the volume of the prescription isodose (60 Gy) and the PTV $\left(\mathrm{V}_{100 \%} / \mathrm{V}_{\text {PTV }}\right)$. For evaluation of the low dose spillage, the ratio of the $50 \%$ isodose volume (30 Gy) and the PTV was calculated $\left(\mathrm{V}_{50 \%} / \mathrm{V}_{\text {PTV }}\right)$. The influence on lung dose was studied by scoring the lung volume receiving 20
Gy, 10 Gy $\left(V_{10 \text { Gy }}\right)$ and the mean lung dose (MLD). For all the other organs at risk, no further analysis was done as these received doses far below the tolerance dose by choosing appropriate beam arrangements. All differences in dosimetric parameters were tested using a paired-sample t-test.

\section{Results \\ Planned dose distributions}

For all three calculation algorithms, clinically acceptable treatment plans could be obtained for all patients (Table 2 ). The dose received by $99 \%$ of the PTV volume is slightly lower for the CC plans (58.5 Gy) compared to the UD (58.8 Gy) and EPL plans (58.8 Gy). Figure 1 shows a boxand-whisker plot of the ratio of the volume of the prescription isodose and the PTV, which is a measure for the conformity of a plan. The conformity for the UD and EPL plans was slightly better compared to the CC plans, although this difference was only statistically significant for the CC and EPL plans. The RTOG criterion $\left(\mathrm{V}_{100 \%} / \mathrm{V}_{\text {PTV }}\right.$ $<1.2$ ) could however be met by all treatment plans except for 3 patients with very small tumors having a minor violation $\left(\mathrm{V}_{100 \%} / \mathrm{V}_{\text {PTV }}<1.4\right)$.

The maximum dose for the CC plans is considerably higher than for the other two (Figure 2). Dose homogeneity in the target area was less for the CC plans, as the effects of tissue inhomogeneities were better accounted for. The broadening of the dose distribution is reflected in the ratio of the volume of the $50 \%$ isodose and the PTV $\left(\mathrm{V}_{50 \%}\right)$ $\mathrm{V}_{\text {PTV }}$ ) which is plotted as a function of the PTV in Figure 3. For the UD and EPL plans, significantly lower ratios were attainable (a mean value of 6.3 for both plans versus 8.4 for the CC plans). Figure 3 also shows that low-dose conformity decreased with decreasing PTV volume, especially for the CC plans.

Regarding the dose to the healthy lung tissue, the $\mathrm{V}_{20 \mathrm{~Gy}}$ for the CC plans was on average $15 \%$ and $21 \%$ higher as the

Table 2: Mean values and the range of dosimetric data for the different treatment plans as planned and recalculated using

\begin{tabular}{|c|c|c|c|c|c|c|c|c|c|}
\hline & \multirow{2}{*}{$\begin{array}{l}\text { CC } \\
\text { Planned }\end{array}$} & \multicolumn{4}{|l|}{ UD } & \multicolumn{4}{|l|}{ EPL } \\
\hline & & Planned & p-value & Recalculated & p-value & Planned & p-value & recalculated & p-value \\
\hline $\mathrm{D}_{95}[\mathrm{~Gy}]$ & 60.0 & 60.0 & - & $56.9[42.1-63.4]$ & 0.007 & 60.0 & - & $48.0[34.6-56.1]$ & $<0.001$ \\
\hline $\mathrm{D}_{99}[\mathrm{~Gy}]$ & 58.5 [57.6-59.5] & $58.8[57.8-59.8]$ & $<0.001$ & $54.5[39.6-62.0]$ & $<0.001$ & $58.8[58.1-59.7]$ & $<0.001$ & $45.9[32.6-53.7]$ & $<0.001$ \\
\hline$D_{\text {isoc }}[G y]$ & $74.3[66.0-84.1]$ & $66.6[62.5-71.3]$ & $<0.001$ & $72.7[59.3-79.6]$ & 0.19 & $67.1[63.2-72.2]$ & $<0.001$ & $61.7[47.9-70.1]$ & $<0.001$ \\
\hline$D_{\text {mean }}[G y]$ & $66.9[64.1-71.0]$ & $64.1[61.6-66.6]$ & $<0.001$ & $65.0[49 .|-7| .0]$ & 0.11 & $64.3[61.8-66.4]$ & $<0.001$ & $55.2[39.7-62.1]$ & $<0.001$ \\
\hline$D_{\max }[G y]$ & $75.6[67.8-87.4]$ & $68.1[63.0-73.3]$ & $<0.001$ & $73.8[6|.3-8| .7]$ & 0.17 & $68.5[63.8-76.5]$ & $<0.001$ & $62.9[48.0-74.2]$ & $<0.001$ \\
\hline$V_{100 \%} / V_{\text {PTV }}$ & $1.13[0.98-1.38]$ & $1.09[0.98-1.25]$ & 0.14 & $0.98[0.01-1.45]$ & 0.08 & $1.09[1.03-1.22]$ & 0.026 & $0.27[0.00-0.7 I]$ & $<0.001$ \\
\hline $\mathrm{V}_{50 \%} / \mathrm{V}_{\mathrm{PTV}}$ & $8.4[4.5-18.2]$ & $6.3[4.4-12.6]$ & $<0.001$ & $6.6[4.9-12.2]$ & 0.006 & $6.3[4.1-12.0]$ & $<0.001$ & $4.8[3.1-6.5]$ & $<0.001$ \\
\hline$V_{20 G y}[\%]$ & $6.6[2.9-16.7]$ & $5.6[2.2-\mid 4.2]$ & $<0.001$ & $6.0[2.3-15.5]$ & 0.017 & $5.2[2.1-12.3]$ & $<0.001$ & $4.1[1.5-10.9]$ & $<0.001$ \\
\hline $\mathrm{V}_{10 \mathrm{~Gy}}[\%]$ & I $3.7[7.3-35.8]$ & $12.1[5.9-29.9]$ & $<0.001$ & $13.1[6.1-33.5]$ & 0.038 & $12.0[5.5-29.5]$ & $<0.001$ & $10.9[4.6-27.3]$ & $<0.001$ \\
\hline MLD [cGy] & $456[257-94 I]$ & $396[203-828]$ & $<0.001$ & $439[223-905]$ & 0.18 & 394 [205-738] & $<0.001$ & $355[183-683]$ & $<0.001$ \\
\hline
\end{tabular}

full inhomogeneity corrections, and the p-values for the paired-sample t-test comparing the UD and EPL calculated and recalculated plans to the CC plan. 


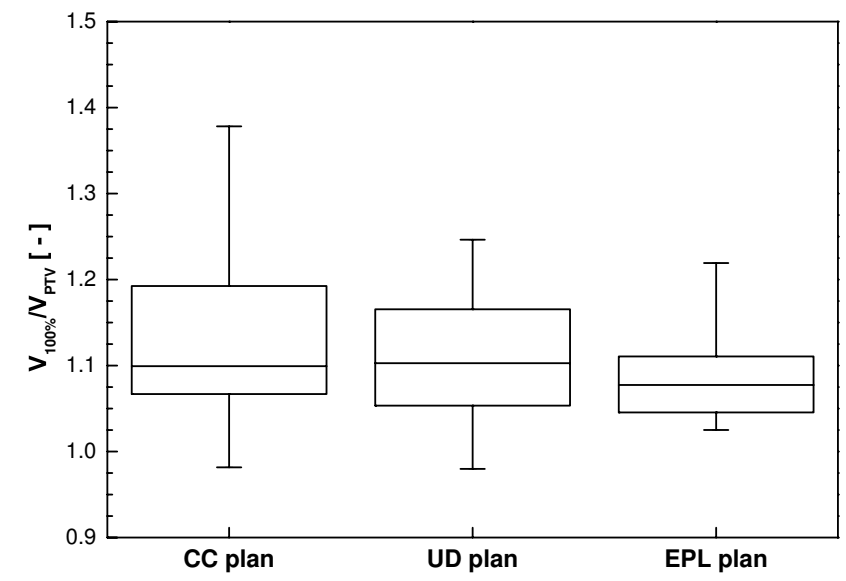

Figure I

Box-and-whisker plot of the conformity index for the optimized plan using full CC calculation, with unit density (UD) and with an equivalent pathlength (EPL) correction.

planned UD and EPL values, respectively (Table 2). For the mean lung dose, the UD and EPL plans both achieved a $13 \%$ lower value compared to the CC plan.

\section{Dose to target and critical organs after recalculation}

The influence of recalculating the UD and EPL plans with the CC algorithm on the dose distribution for an individual patient is illustrated in Figure 4 . The shape of the dose distribution changed significantly, leading to unwanted high dose regions, possibly endangering critical structures like the ribs or hilar vessels.

The dose covering 95\% of the PTV for the recalculated plans as a function of the PTV is plotted in Figure 5. The EPL plans consistently overestimate the dose to the PTV, resulting in an average $\mathrm{D}_{95}$ of $48 \mathrm{~Gy}, 20 \%$ lower than the prescribed value. For one patient, a dose of $35 \mathrm{~Gy}$ was even observed, 43\% lower than planned. The overestimation of the dose increased with decreasing PTV size, although large variations are observed between individual patients. For the UD calculations the recalculated plans on average had a slightly lower $\mathrm{D}_{95}$ of $57 \mathrm{~Gy}$, with values ranging between 63 and $42 \mathrm{~Gy}$ for individual patients. No correlation was found with PTV size. For the other dosimetric parameters $\left(D_{99}, D_{\text {isoc, }}, D_{\text {mean }}\right)$ similar results are found as for $\mathrm{D}_{95}$ (Table 2). For the maximum dose, larger values were found for the recalculated plans (Figure 6).

Changes in PTV coverage were also reflected in $\mathrm{V}_{100 \%} / \mathrm{V}_{\text {PTV }}$ (Figure 7), with a significant decrease of this ratio for the EPL plans and also a small though not statistically significant decrease for the UD plans. Again, large variations

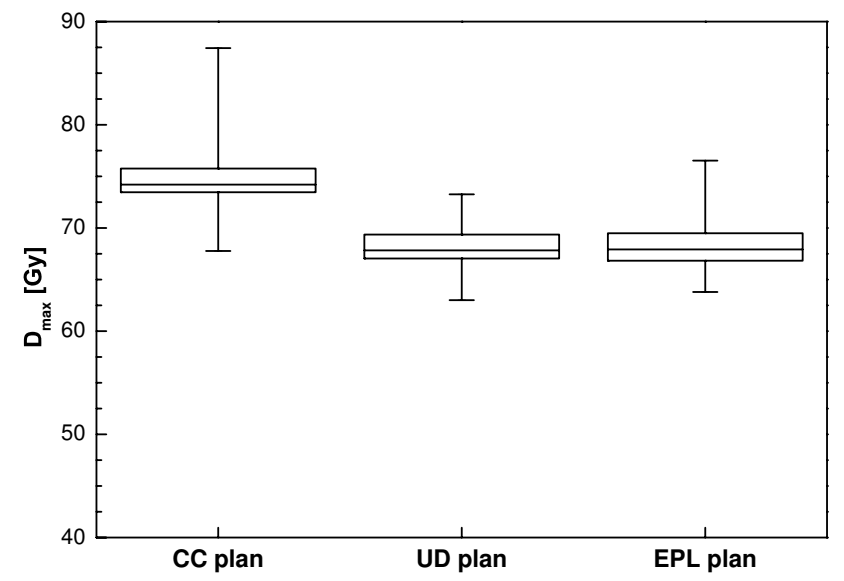

Figure 2

Box-and-whisker plot of the maximum dose for the optimized plans before recalculation.

between patients were especially visible for the UD plans, with ratios ranging from 0.01 to 1.45 .

For the recalculated UD plans, the mean $\mathrm{V}_{20 \mathrm{~Gy}}$ was significantly different from the CC plans ( 6.0 versus $6.6 \%$ ), and large variations per patient existed. For the EPL plans the mean $\mathrm{V}_{20 \text { Gy }}$ was significantly lower, with a mean value of $4.1 \%$. The recalculated $V_{20}$ Gy is plotted against the planned $\mathrm{V}_{20 \mathrm{~Gy}}$ for the UD and EPL plans in Figure 8. These plots were fitted using linear regression, the resulting fit parameters can be found in table 3. A strong dependency existed between planned and recalculated values $\left(\mathrm{R}^{2}=\right.$ 0.99 and 0.96 for UD and EPL plans, respectively)

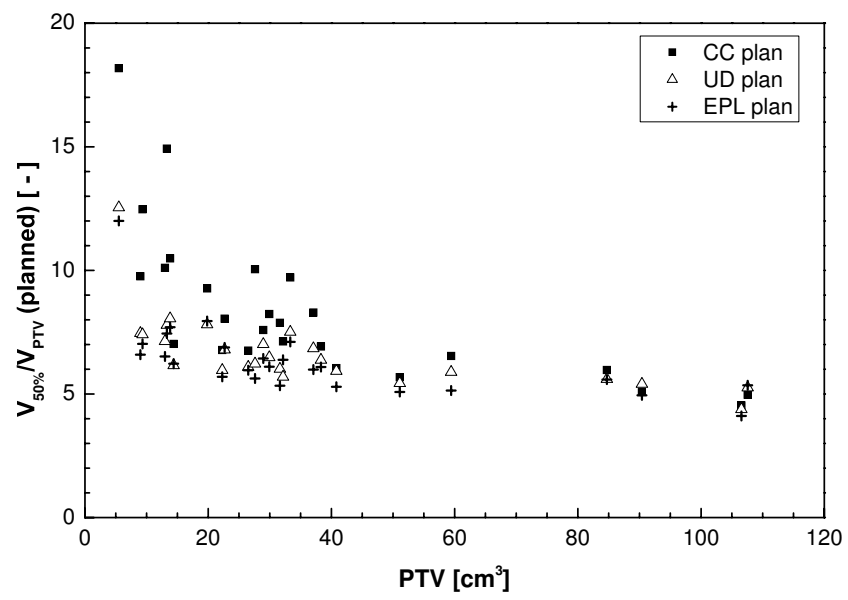

Figure 3

Ratio of the volume of the $50 \%$ isodose and the PTV as a function of PTV for the three plans before recalculation. 


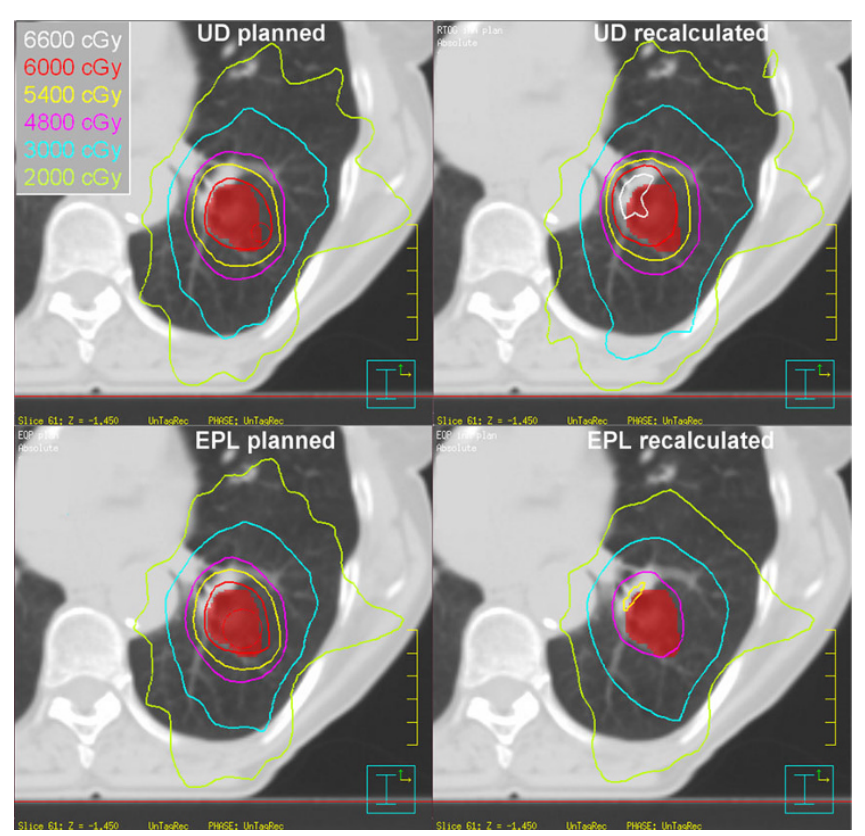

Figure 4

Example of the planned and recalculated dose distribution for the UD (top) and EPL (bottom) calculation for a single patient. Note the much higher dose to the hilar structure in the recalculated UD plan and the much lower dose coverage in the recalculated EPL plan.

although a reasonable amount of scatter is visible. For the $\mathrm{V}_{10 \mathrm{~Gy}}$ and mean lung dose even stronger correlations were found between planned and recalculated values (Table 3 ). The recalculated $\mathrm{V}_{10 \mathrm{~Gy}}$ was about $8 \%$ higher for the UD plans, and $8 \%$ lower for the EPL plans, for the MLD an $11 \%$ increase and $10 \%$ decrease was found, respectively.

\section{Discussion}

In this study it was demonstrated that the use of different inhomogeneity corrections during the planning of stereotactic lung RT treatments has a large impact on the dose distribution to the target area and healthy lung tissue. The separation into two types of algorithms ( $a$ and $b$ ) as mentioned in the introduction is of course a simplification of the differences that exist between the various clinically implemented algorithms. The comparison between the EPL and CC algorithms presented here can be seen as a good quantitative analysis of the differences that can be found between type a and b algorithms. However, slightly different results are expected if two other (implementations of) type $\mathrm{a}$ and $\mathrm{b}$ algorithms would have been used.

The dose criteria as prescribed in the RTOG 0236 trial [4] have been based on calculations assuming unit density of the patient. For the algorithms accounting for the effect of the increased lateral range of the electrons and scattered

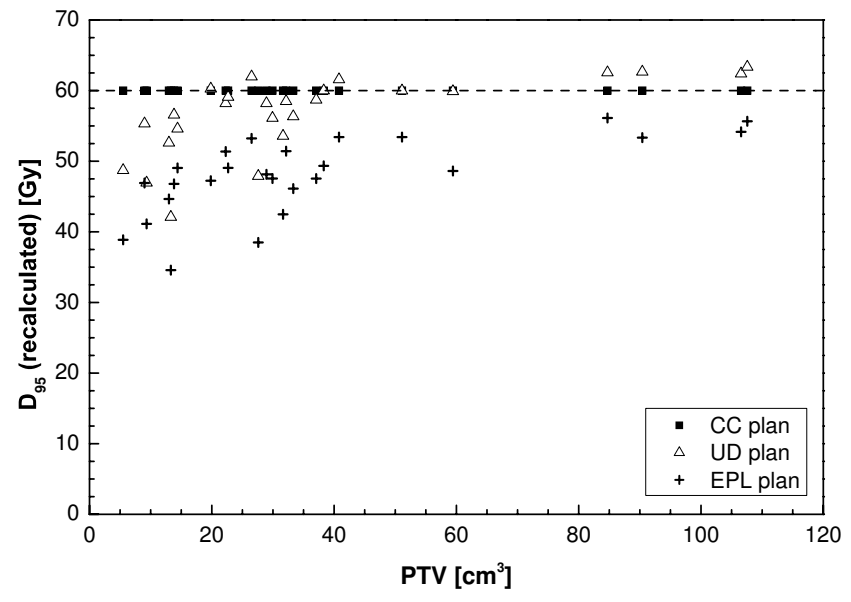

Figure 5

Dose to $\mathbf{9 5 \%}$ of the PTV as a function of the PTV as determined using the $\mathrm{CC}$ calculation.

photons, these criteria are often harder to fulfill as the penumbras tend to be broadened.

Dose coverage of the recalculated plans varied widely among different patients. The dose to $95 \%$ of the PTV for the plans optimized with unit density ranged from $30 \%$ lower to slightly higher than planned for individual patients. A simple rescaling of the planned dose to the actual dose given to the patient is thus not possible, making a recalculation of the plan with accurate dose calculations necessary.

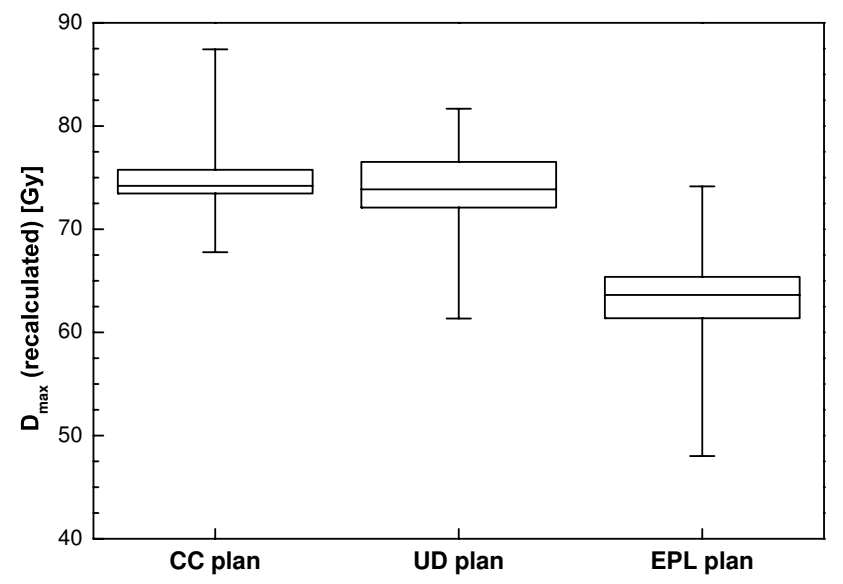

Figure 6

Box-and-whisker plot of the maximum dose after recalculation of the unit density and EPL plan. The results from the $\mathrm{CC}$ plan are plotted for comparison. 


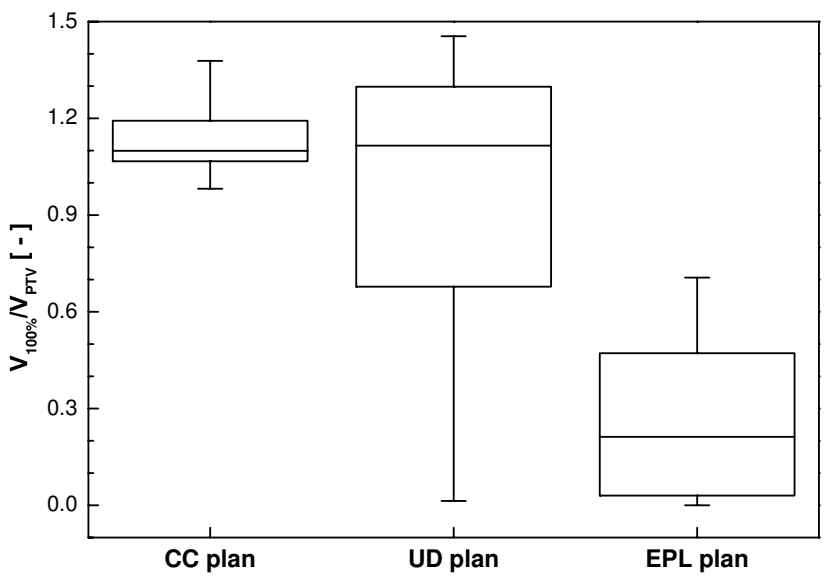

Figure 7

Box-and-whisker plot of the conformity index for the UD and EPL plans after recalculation with full inhomogeneity corrections. The results from the collapsedcone calculation are plotted for comparison.

The overestimation of the dose using the EPL algorithm seen in all patients varies with increases tumorsize, lung density and location. This type of algorithm is still widely used in clinical practice, and has also been used by Lagerwaard et al. who recently presented clinical results of more than 200 patients with NSCLC [3]. The possible overestimation of the dose given to the tumor should be considered when interpreting the results from this clinical study.

In a study by Haedinger et al.[12] the stereotactic treatments from 33 lung cancer patients planned with a pencil beam algorithm were recalculated using a CC algorithm. These authors found a smaller overestimation of the dose given to the target using a pencil beam algorithm. This might in part be explained by the planning itself, as the PTV coverage in their work was often higher than the 95\% used in this study. Another important difference is the PTV sizes considered in their study, with a median value of 122 cc compared to 29 cc in our study, which is more representative for patients suitable for this treatment. As a result smaller fieldsizes were used in our study, resulting in a larger overestimation of the dose.

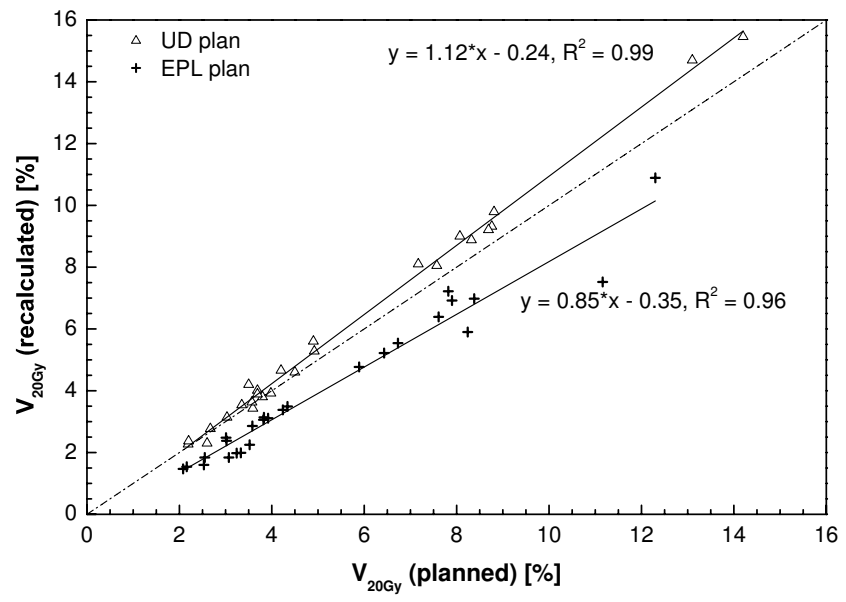

Figure 8

Recalculated lung volume receiving 20 Gy or more versus planned value for the unit density and EPL plans.

As the UD and EPL calculations do not account for the increased lateral electron range, recalculation of the plans results in an increase of the low-dose region $\left(\mathrm{V}_{20 \mathrm{~Gy}}, \mathrm{~V}_{10 \mathrm{~Gy}}\right)$ in the lungs. On the other hand the algorithms often underestimate the required number of MUs due to lateral electron disequilibrium. As a result, the plans optimized with unit density calculations tend to underestimate the dose to the healthy lung $\left(\mathrm{V}_{20 \mathrm{~Gy}}, \mathrm{~V}_{10 \mathrm{~Gy}}\right.$ and MLD), and the EPL plans overestimate it. In accordance with De Jaeger et al. [15] a correlation was found between planned and recalculated values for the lung dose. As this study deals with much smaller target volumes compared to the ones used by De Jaeger, lateral electron disequilibrium is more dominant, resulting in different correlations than the ones found by these authors. In contrast, Chang et al did not find a difference between V20 Gy values calculated for heterogeneity corrected and UD plans [16]. However, they predominately used simple anteroposterior/posterioranterior fields and irradiated much larger lung volumes, as can be derived from their high V20 values in combination with their dose prescriptions. This again might explain the difference found between our results, using stereotactic radiotherapy to treat relatively small target volumes and the results derived by Chang et al. With yet another beam set-up, namely for breast irradiation, Brink

Table 3: Correlation between planned and recalculated values for the lung volume receiving 20 Gy, 10 Gy and the mean lung dose.

\begin{tabular}{lllll}
\hline Parameter & $\begin{array}{l}\text { Unit density } \\
\text { Correlation }\end{array}$ & $\mathbf{R}^{2}$-value & $\begin{array}{l}\text { EPL } \\
\text { Correlation }\end{array}$ & $\mathbf{R}^{2}$-value \\
\hline $\mathrm{V}_{20 \mathrm{~Gy}}$ & $1.12 \cdot \mathrm{V}_{20 \mathrm{~Gy}, \text { planned }}-0.24$ & 0.994 & $0.85 \cdot \mathrm{V}_{20 \mathrm{~Gy} \text {, planned }}-0.35$ & 0.957 \\
$\mathrm{~V}_{10 \mathrm{~Gy}}$ & $1.08 \cdot \mathrm{V}_{10 \mathrm{~Gy} \text {, planned }}-0.05$ & 0.996 & $0.92 \cdot \mathrm{V}_{10 \mathrm{~Gy} \text {, planned }}-0.07$ & 0.979 \\
$\mathrm{MLD}$ & $1.11 \cdot \mathrm{MLD}_{\text {planned }}-$ & 0.998 & $0.90 \cdot \mathrm{MLD}_{\text {planned }}$ & 0.999 \\
\hline
\end{tabular}


et al. also found significant differences between algorithms in deriving optimal radiation pneumonitis NTCP values [17]. Thus, it might be concluded that differences between algorithms in calculating lung dose do exist. The extent of the deviation depends on the algorithms and irradiation techniques investigated.

Although Monte Carlo simulations are considered to be the gold standard in the presence of inhomogeneities, the collapsed cone algorithm has proven to be reasonably accurate. Krieger and Sauer did find up to $10 \%$ difference between CC and Monte-carlo calculations. However, these deviations were found using a slab geometry phantom and single beam set-up which does not resemble a clinical set-up very well. Furthermore, the authors indicate that part of these errors might be explained by an incorrect choice of the CC parameters [9]. In most situations, accuracy in the order of 2 to $5 \%$ is obtainable, meaning that inaccuracies in the CC dose calculations are much smaller than the differences observed in this study, and can be considered as a reasonably accurate representation of the actual dose given to the patient $[18,17,10]$.

Maybe even more important, the collapsed cone algorithms have now become widely available in clinical practice, while the use of Monte Carlo treatment planning is still very limited. Thus, the CC results presented here can be used to generate optimization criteria in clinical practice, while this would be less straightforward for results based on Monte-Carlo calculations.

\section{Conclusion}

The implications of the results in this study are twofold. In the first place, planning dose criteria are often easier achieved with plans created using simple dose calculation algorithms, which should be considered in study designs involving multiple institutions using different planning systems. Secondly, the actually delivered dose to the tumor can significantly deviate from the planned value when not using appropriate inhomogeneity corrections. As large variations exist in the actual dose per individual patient, clinical studies evaluating the effectiveness of this treatment should rely on the most accurate dose calculation which is clinically available at the time, or at least retrospectively re-evaluate the actual dose given to the target. Fortunately, the dose to the healthy lung tissue calculated with a simple algorithm can retrospectively easily be recalculated using the correlation parameters derived in this study. Before clinical introduction, the fractionation scheme and dose optimization procedure should be very well tailored to the calculation algorithm and TPS one uses clinically.

\section{Authors' contributions}

DS was responsible for study design, carried out treatment planning, analysis of data and results, and writing and editing the manuscript, CWH worked on study design, analysis of data and results, and writing and editing the manuscript. All authors have read and approved the final manuscript.

\section{Acknowledgements}

The authors would like to thank Marjan Admiraal and Gert Meijer for fruitful discussions. Philips Medical Systems is gratefully acknowledged for providing some of the 4D-CT capabilities within the context of a research agreement. Part of this work has been presented at the annual ESTRO meeting in 2007.

\section{References}

I. Onishi H, Shirato H, Nagata $Y$, Hiraoka M, Fujino M, Gomi K, Niibe Y, Karasawa K, Hayakawa K, Takai Y, Kimura T, Takeda A, Ouchi A, Hareyama M, Kokubo M, Hara R, Itami J, Yamada K, Araki T: Hypofractionated stereotactic radiotherapy (HypoFXSRT) for stage I non-small cell lung cancer: updated results of 257 patients in a Japanese multi-institutional study. J Thorac Oncol 2007, 2:S94-100.

2. Timmerman R, Papiez L, McGarry, Likes L, DesRosiers C, Frost S, Williams M: Extracranial stereotactic radioablation - results of a phase I study in medically inoperable stage I non-small cell lung cancer. Chest 2003, I 24:1946-1955.

3. Lagerwaard FJ, Haasbeek CJA, Smit EF, Slotman BJ, Senan S: Outcomes of risk-adapted fractionated stereotactic radiotherapy for stage I non-small-cell lung cancer. Int J Radiat Oncol Biol Phys 2008, 70:685-692 [http://linkinghub.elsevier.com/retrieve/pii/ S0360301607044689].

4. Timmerman RD, Michalski J, Galvin J, Fowler JF, Choy H, gore E, johnstone D: RTOG 0236: A phase II trial of stereotactic body radiation therapy (SBRT) in the treatment of patients with medically inoperable stage I/II non-small cell lung cancer. RTOG; 2006.

5. Nishio T, Kunieda E, Shirato H, Ishikura S, Onishi H, Tateoka K, Hiraoka M, Narita Y, Ikeda M, Goka T: Dosimetric verification in participating institutions in a stereotactic body radiotherapy trial for stage I non-small cell lung cancer: Japan clinical oncology group trial (JCOG0403). Phys Med Biol 2006, 5 I:5409-54I7.

6. Knöös T, Wieslander E, Cozzi L, Brink C, Fogliata A, Albers D, Nyström H, Lassen S: Comparison of dose calculation algorithms for treatment planning in external photon beam therapy for clinical situations. Phys Med Biol 2006, 5 I:5785-5807.

7. Lax I, Panettieri V, Wennberg B, Amor DM, Naslund I, Baumann P, Gagliardi G: Dose distributions in SBRT of lung tumors: Comparison between two different treatment planning algorithms and Monte-Carlo simulation including breathing motions. Acta Oncol 2006, 45:978-988.

8. Jones $A O$, Das I: Comparison of inhomogeneity correction algorithms in small photon fields. Med Phys 2005, 32:766-776.

9. Krieger T, Sauer OA: Monte Carlo- versus pencil-beam-/collapsed-cone-dose calculation in a heterogeneous multi-layer phantom. Phys Med Biol 2005, 50:859-868.

10. Fogliata A, Nicolini G, Vanetti E, Clivio A, Winkler P, Cozzi L: The impact of photon dose calculation algorithms on expected dose distributions in lungs under different respiratory phases. Phys Med Biol 2008, 53:2375-2390.

II. Ding GX, Duggan DM, Lu B, Hallahan DE, Cmelak A, Malcolm A, Newton J, Deeley M, Coffey CW: Impact of inhomogeneity corrections on dose coverage in the treatment of lung cancer using stereotactic body radiation therapy. Med Phys 2007, 34:2985-2994.

12. Haedinger U, Krieger T, Flentje M, Wulf J: Influence of calculation model on dose distribution in stereotactic radiotherapy for pulmonary targets. Int J Radiat Oncol Biol Phys 2005, 6 I :239-249.

13. Panettieri V, Wennberg B, Gagliardi G, Duch MA, Ginjaume M, Lax I: SBRT of lung tumours: Monte Carlo simulation with PENELOPE of dose distributions including respiratory motion and comparison with different treatment planning systems. Phys Med Biol 2007, 52:4265-428I.

14. Hansen AT, Petersen JB, Hoyer M, Christensen JJ: Comparison of two dose calculation methods applied to extracranial stere- 
otactic radiotherapy treatment planning. Radiother Oncol 2005, 77:96-98.

15. de Jaeger K, Hoogeman MS, Engelsman M, Seppenwoolde Y, Damen EFM, Mijnheer BJ, Boersma LJ, Lebesque JV: Incorporating an improved dose-calculation algorithm in conformal radiotherapy of lung cancer: re-evaluation of dose in normal lung tissue. Radiother Oncol 2003, 69:I-I0.

16. Chang DT, Olivier KR, Morris CG, Liu C, Dempsey JF, Benda RK, Palta JR: The impact of heterogeneity correction on dosimetric parameters that predict for radiation pneumonitis. Int J Radiat Oncol Biol Phys 2006, 65: I25-I3I.

17. Brink C, Berg M, Nielsen M: Sensitivity of NTCP parameter values against a change of dose calculation algorithm. Med Phys 2007, 34:3579-3586.

18. Vanderstraeten B, Reynaert N, Paelinck L, Madani I, de Wagter C, de Gersem W, de Neve W, Thierens H: Accuracy of patient dose calculation for lung IMRT: A comparison of Monte Carlo, convolution/superposition, and pencil beam computations. Med Phys 2006, 33:3149-3158.

Publish with Bio Med Central and every scientist can read your work free of charge

"BioMed Central will be the most significant development for disseminating the results of biomedical research in our lifetime. "

Sir Paul Nurse, Cancer Research UK

Your research papers will be:

- available free of charge to the entire biomedical community

- peer reviewed and published immediately upon acceptance

- cited in PubMed and archived on PubMed Central

- yours - you keep the copyright

Submit your manuscript here:

http://www.biomedcentral.com/info/publishing_adv.asp 